\title{
Photoresponse of Porous Silicon Structures to Infrared Radiation
}

\author{
N. Samuolienti ${ }^{a}, *$ E. Širmulis ${ }^{b}$, J. Stupakova $^{a}$, J. Gradauskas $^{a}$, \\ V. ZAGADSKIJ ${ }^{a}$ AND E. ŚATKOVSKIS ${ }^{a}$ \\ ${ }^{a}$ Vilnius Gediminas Technical University, Sauletekio ave. 11, Vilnius LT-10223, Lithuania \\ ${ }^{b}$ Centre for Physical Science and Technology, Semiconductor Physics Institute
}

A. Goštauto 11, Vilnius LT-01108, Lithuania

\begin{abstract}
Photoresponse of silicon samples containing porous structures have been studied under the action of $\mathrm{CO}_{2}$ laser radiation. The signal shape and its behavior under the applied bias voltage revealed the existence of two heterojunctions on the border of porous-crystalline silicon and on the border between the porous layers of different porosity. The photosignal is recognized to be composed of hot hole photoemfs induced across the heterojunctions.
\end{abstract}

PACS: 78.67.Rb, 78.56.-a, 73.40.Lq

\section{Introduction}

Porous silicon draws attention of researchers because of its unusual properties that are absent in a crystalline silicon (c-Si), due to relative simplicity and availability of technology, and because of unexpected new possibilities of applications. Porous silicon technology enables to create silicon nanostructures which are advanced objects of modern research. In addition, this way of production of nanostructures does not demand high expenses.

For the first time manufacturing of quantum wires in porous silicon was declared by Canham [1] and by Lehmann and Gösele [2]. These works served as a powerful breakthrough to the development of nanotechnology of porous silicon and to intensive research of silicon quantum structures, silicon quantum wires and silicon quantum dots, thus releasing from necessity to use technically more perfect but more expensive modern technologies. Strong stimulus for the investigations was discovery of changes of forbidden energy gap resulting in strong increase of radiating recombination efficiency in silicon quantum structures [3]. This opening released silicon from reputation of badly radiating semiconductor. Effective photoluminescence in porous silicon in a couple with the effect of dimensional quantum confinement both anticipated attempts to make an effective silicon source of spontaneous radiation or even a silicon laser to a reality. Considerable quantity of interesting works was devoted to create a silicon radiator by means of cheap porous silicon technology $[4,5]$. A series of recent works was executed on the research of influence of microwave radiation

\footnotetext{
* corresponding author; e-mail: eusat@vgtu.lt
}

on the properties of porous silicon structures [6,7]. It was revealed that the structures had by several orders higher sensitivity to microwave radiation than similar semiconductor structures without a porous layer. The results were explained within the model of charge carrier heating in the structure containing a layer of microporous silicon with typical quantum confinement properties [8]. According to the model, two heterojunctions were formed in the structure because of different width of the forbidden energy gaps of the crystalline and microporous silicon. Heating of charge carriers by a microwave radiation induced rise of an electromotive force (emf) across the junction. Intense infrared radiation may also initiate heating of charge carriers. Formation of hot carrier emf in crystalline semiconductor structures have been investigated widely $[9,10]$, still no such results are reported on a porous silicon.

Characteristics of time-dependence of a photoresponse and its behavior under the action of external fields can give information about physical processes running in the sample. In this paper we use analysis of the shape of the induced photoresponse pulses under various conditions and investigate its formation in the structure containing a layer of microporous silicon. $\mathrm{A} \mathrm{CO}_{2}$ laser served as an infrared radiation source.

\section{Experimental}

Specimens containing porous silicon structure were manufactured of monocrystalline $p$-type silicon plates of resistivity $0.4 \Omega \mathrm{cm}$ and crystal orientation (100). Porous silicon layers were produced by an electrochemical etching method. Mix of fluoric acid and ethanol in the ratio 1:2 was used as an electrolyte. The structures containing 
two porous silicon layers PSiL1 and PSiL2 were prepared. Full cycle of etching consisted of two phases: the density of anodic current was $10 \mathrm{~mA} / \mathrm{cm}^{2}$ and $80 \mathrm{~mA} / \mathrm{cm}^{2}$, and etching time was 5 and $10 \mathrm{~min}$, respectively, for each phase. Denser auxiliary PSiL1 layer was formed at the contact during the first phase. Porosity of the auxiliary layer was estimated to be about $55 \%$, and its thickness was about $3-5 \mu \mathrm{m}$. The main layer of microporous silicon (PSiL2) was formed in the second phase. Its thickness was about $20 \mu \mathrm{m}$, and porosity reached about $75 \%$ (Fig. 1). Pulsed $\mathrm{CO}_{2}$ laser radiation of $10.6 \mu \mathrm{m}$ wavelength was used to excite the samples. Duration of the pulses was $150 \mathrm{~ns}$, pulse power reached $100 \mathrm{~kW}$. The laser pulses were controlled by a cooled $\mathrm{Ge}(\mathrm{Au})$ photodetector. Induced electrical pulses were registered by an oscilloscope LeCroy Wavepro 7200 with $2 \mathrm{GHz}$ passband. Experiment was carried out at room temperature.

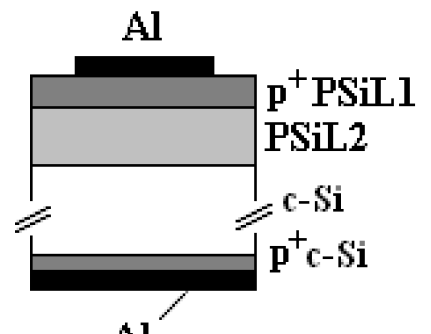

Al

Fig. 1. Schematic drawing of the structure containing porous silicon layers with additionally boron doped top and bottom sides: $\mathrm{Al}$ - aluminum contacts, $p^{+}$PSiL1 — porous silicon layer of $55 \%$ porosity, PSiL2 porous silicon layer of $75 \%$ porosity, $\mathrm{c}-\mathrm{Si}$ and $p^{+} \mathrm{c}-\mathrm{Si}$ - monocrystalline silicon plate and its doped layer, respectively.

\section{Results and discussion}

Typical traces of photoresponse arising across the fabricated samples exposed to the $\mathrm{CO}_{2}$ laser radiation are shown in Fig. 2. The laser pulse traced by a $\mathrm{Ge}(\mathrm{Au})$ photodetector is presented there as well.

The leading front of the photoemf is fast and almost follows the shape of the laser pulse, only the signal reaches its maximum value slightly later in respect of the laser pulse. Application of negative bias to the porous silicon layer (in point of positive potential applied to the $\mathrm{c}-\mathrm{Si}$ ) gives rise to the signal amplitude herewith keeping the time parameters of the photoresponse respectively unchanged (Fig. 2, trace 2). Different picture is observed when the positive bias voltage is applied. Now not only the amplitude of the photoresponse decreases, but also the shape of the pulse is changed with the appearance of the component of opposite polarity at the leading front of the pulse (Fig. 2, trace 3).

It is worth noting that in all three cases the photosignal reaches maximum value well before the end of the laser pulse; thereby it means that the signal rise is not initiated

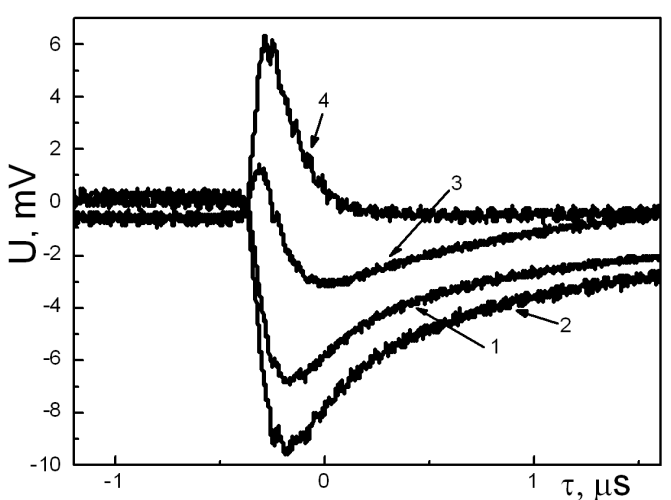

Fig. 2. Photoresponse of the structures containing porous silicon: 1 - no bias, 2 - negative bias on the porous silicon layer, 3 - positive bias on the porous silicon layer, $4-\mathrm{CO}_{2}$ laser pulse shape. Bias voltage is $1.5 \mathrm{~V}$, load resistance $50 \Omega$.

by a simple heating of the sample. On the other hand, the photon energy of $10.6 \mu \mathrm{m}$ wavelength radiation is insufficient to cause interband excitation of charge carriers in silicon. Therefore, the observed experimental results may be discussed within the limits of the model of free holes' heating by the $\mathrm{CO}_{2}$ laser radiation, and the detected photosignal may be treated as a hot carrier emf arising in the structure. Since hot carrier effects are very fast (the rate is mainly determined by the carrier energy relaxation time), the proposed model is strongly maintained by the experimental fact showing short subpulses on the photosignal perfectly repeating the subpulses of the laser pulse; one of them is well seen on the very peak of the pulse. Free carrier absorption is mainly responsible for the absorption of a $10.6 \mu \mathrm{m}$ wavelength laser radiation in silicon at room temperature $[10,11]$. In the case of $p-n, l-h$ junction or other kind of potential barrier, a hot carrier emf arises across the inhomogeneity exposed to an intensive $\mathrm{CO}_{2}$ laser radiation $[9,10]$. The polarity of the emf indicates flow of the heated carriers over a barrier.

The microcrystalline PSiL2 silicon layer should be in a state of quantum confinement since its porosity reaches $75 \%[3,7,8]$, whereas no quantum confinement effect should be present in the nearby layers of denser PSiL1 and c-Si. Consequently, the forbidden energy gap of the mid layer should be wider than that of the neighboring layers. Thus, we can state that two heterojunctions are formed on the both borders of the porous microcrystalline silicon layer. Schematic energy band diagram of the $p$-type silicon structure is presented in Fig. 3 . The microcrystalline silicon PSiL2 layer of wider forbidden energy gap $E_{\mathrm{g} 2}$ is sandwiched between the two layers of narrower $E_{\mathrm{g}}$. The potential barrier heights of both heterojunctions should be almost equal. Still there exists possibility for the PSiL1/PSiL2 barrier to be some higher due to additional boron-doping performed during the contact preparation. 


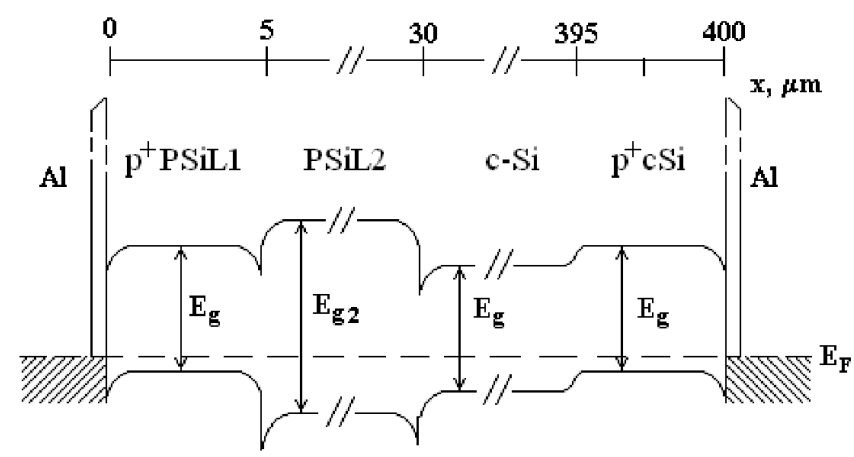

Fig. 3. Scheme of energy bands across the sample. $E_{\mathrm{g}}$ and $E_{\mathrm{g} 2}$ denote forbidden energy gaps.

Illumination of the structure with the $\mathrm{CO}_{2}$ laser radiation stimulates rise of photosignal with polarity correspondent to the flow of the holes from the right side to the left side, according to Fig. 3. Taking into account the hot carrier model, two hot hole photoemfs of opposite polarity should be induced across the both heterojunctions simultaneously. Since the absorption coefficient of free holes in silicon does not exceed $10^{3} \mathrm{~cm}^{-1}$ in the vicinity of $10 \mu \mathrm{m}$ wavelength, and the sample was irradiated from the porous layer side, the radiation density falling over the both heterojunctions is considered to be the same. But since the c-Si layer is denser than the porous PSiL1 where the radiation is absorbed comparatively poorly, and since the potential barrier of the PSiL2/c-Si junction may be lower than the other one, most probably the detected photosignal is composed mainly of the strong dominating photoemf arising across the PSiL2/c-Si heterojunction and weak negligible photoemf arising across the PSiL1/PSiL2 heterojunction. The signal polarity supports this contemplation.

Application of the negative bias voltage (minus sign on the left of Fig. 3) lowers the potential barrier of the right-side heterojunction thus stimulating increased flow of the heated holes over the barrier, i.e. the photoemf across the PSiL2/c-Si junction is increased (see Fig. 2, trace 2). The PSiL1/PSiL2 potential barrier is heightened in this case.

Positive bias voltage forms opposite situation. The PSiL1/PSiL2 potential barrier height is lowered, and the
PSiL2/c-Si barrier is increased. As trace 3 in Fig. 2 shows, in this case the hot carrier photoemf induced across the PSiL1/PSiL2 heterojunction tends to dominate among the components of the photosignal.

\section{Conclusion}

Investigation of porous silicon structures manufactured of monocrystalline $p$-type silicon and exposed to infrared $\mathrm{CO}_{2}$ laser radiation revealed the existence of two heterojunctions: the one on the border of the microporous silicon with crystalline silicon, and the other on the border with silicon of lower porosity. Formation of the heterojunctions results from forbidden energy gap difference caused by a quantum confinement effect. Hot carrier effect is responsible for the rise of emfs across both heterojunctions.

\section{References}

[1] L.T. Canham, Appl. Phys. Lett. 57, 1046 (1990).

[2] V. Lehmann, U. Gösele, Appl. Phys. Lett. 58, 856 (1991).

[3] S. Gardellis, J.S. Rimmer, P. Dawson, B. Hamilton, R.A. Kubiak, T.E. Whall, E.H.C. Parker, Appl. Phys. Lett. 59, 2118 (1991).

[4] L. Pavesi, Riv. Nuove Cimento 20, 1 (1997).

[5] P.M. Fauchet, J. Lumin. 70, 294 (1996).

[6] E. Shatkovskis, J. Gradauskas, J. Stupakova, A. Česnys, A. Sužiedèlis, Lith. J. Phys. 47, 169 (2007).

[7] J. Stupakova, S. Ašmontas, J. Gradauskas, V. Zagadskij, E. Shatkovskis, A. Sužiedèlis, Acta Phys. Pol. A 110, 817 (2006).

[8] E. Shatkovskis, J. Gradauskas, A. Česnys, J. Stupakova, A. Sužiedèlis, Acta Phys. Pol. A 113, 993 (2008).

[9] S. Ashmontas, E. Shirmulis, Fiz. Tekh. Poluprovodn. 20, 2212 (1985) [Sov. Phys.-Semicond. 20, 1382 (1986)].

[10] S. Ashmontas, J. Gradauskas, E. Shirmulis, Sov. Phys.-Semicond. 27, 653 (1993).

[11] A. Dargys, J. Kundrotas, Handbook on Physical Properties of Ge, Si, GaAs and InP, Science and Encyclopedia Pub., Vilnius 1994. 